\title{
Adaptação e Validação Transcultural de uma Medida de Fatores de Absenteísmo
}

\author{
Sinésio Gomide Junior ${ }^{1}$ \\ Marco Aurélio Silva Esteves ${ }^{2}$ \\ Ligia Carolina Oliveira Silva ${ }^{1}$ \\ ${ }^{1}$ Universidade Federal de Uberlândia (Uberlândia-MG) \\ ${ }^{2}$ Faculdade Serra da Mesa (Uruacu-GO)
}

\begin{abstract}
Resumo
O absenteísmo laboral, fenômeno complexo, é caracterizado pela ausência de um empregado de suas atividades trabalhistas. Apesar da importância do tema, visto que pode provocar impactos na produtividade e nos custos de uma organização, não foram encontrados instrumentos sobre o absenteísmo laboral validados para a população brasileira. O objetivo deste trabalho foi realizar uma adaptação e validação transcultural de uma escala de absenteísmo laboral. O trabalho foi constituído por dois estudos. No primeiro, foi realizada a adaptação semântica e análise fatorial exploratória do instrumento. No segundo estudo, foi realizada a análise fatorial confirmatória a partir dos resultados obtidos no primeiro estudo. Os resultados obtidos confirmam a validade do instrumento adaptado que se apresentou como unifatorial, com precisão bastante satisfatória $(\alpha=0,91)$. Os resultados são discutidos a partir das concepções teóricas do construto, da possibilidade de sua utilização em diagnósticos e pesquisas de cunho acadêmico e das limitações dos estudos.
\end{abstract}

Palavras-chave: absenteísmo laboral, adaptação e validação de instrumento, psicometria

Transcultural adaptation and validation of factors within absenteeism

\begin{abstract}
The complex phenomenon known as labor absenteeism is characterized by the absence of an employee from his or her work duties. Despite the importance of this theme, given the possible impact it may have on productivity and costs of an organization, no validated instruments on labor absenteeism were found for the Brazilian population. The objective of the present work was to conduct a transcultural adaptation and validation of a labor absenteeism scale. The work consisted of two studies. The first focused on achieving a semantic adaptation of the original instrument to Brazilian Portuguese and exploratory factor analysis. In the second study a confirmatory factor analysis was performed based on the results of the first study. The results confirmed the validity of the adapted instrument, which presented itself as unifactorial, with very satisfactory precision $(\alpha=$ 0.91). The results are discussed based on the theoretical conceptions of the construct, the possibility of its use in diagnostic and academic research, and the limitations of the studies.

Keywords: labor absenteeism; instrument adaptation and validation; psychometrics
\end{abstract}

Adaptación y Validación Transcultural de una Medida de los Factores de Absentismo

\section{Resumen}

El absentismo laboral, fenómeno complejo, se caracteriza por la ausencia de un empleado en sus actividades laborales. A pesar de la importancia del tema, ya que puede provocar impactos en la productividad y en los costos de una organización, no se encontraron instrumentos validados de absentismo laboral para la población brasileña. El objetivo de este estudio fue realizar una adaptación y validación transcultural de una escala de absentismo laboral. El trabajo fue constituído por dos estudios. En el primer estudio se realizó la adaptación semántica y el análisis factorial exploratorio del instrumento. En el segundo estudio, se realizó análisis factorial confirmatorio a partir de los resultados obtenidos en el primer estudio. Los resultados obtenidos confirman la validez del instrumento adaptado que se presentó como unifactorial, con precisión bastante satisfactoria $(\alpha=0,91)$. Los resultados son discutidos a partir de las concepciones teóricas del constructo, de la posibilidad de su utilización en diagnósticos e investigaciones de índole académico y de las limitaciones de los estudios.

Palabras clave: absentismo laboral; adaptación y validación de instrumento; psicometría

A produtividade e o bem-estar dos trabalhadores de uma organização podem ser influenciados por distintos fatores, dentre eles, o absenteísmo laboral que causa impacto significativo nos resultados de uma organização (Jesus \& Murcho, 2014). Segundo a Organização Internacional do Trabalho - OIT (1989), o absenteísmo laboral é caracterizado como a falta ao trabalho por parte do empregado. De acordo com Jesus e Murcho (2014), o absenteísmo pode ser qualificado em quatro tipos distintos. O primeiro, voluntário, é ocasionado por questões particulares dos trabalhadores, não havendo qualquer justificativa formal diante da organização. O segundo, por doença, ocorre quando é causado por patologias ou procedimentos médicos que não estão relacionados ao trabalho. $\mathrm{O}$ terceiro, identificado como "acidentes" ou "doenças trabalhistas" é 
aquele que decorre da atuação profissional do trabalhador e, por último, o compulsório, quando decorre de sanções disciplinares da organização ou a prisão do trabalhador (Jesus \& Murcho, 2014).

O absenteísmo laboral é entendido como um fenômeno complexo devido à diversidade de elementos que podem provocá-lo. Calheiros e Rodrigues (2010) destacam a comunicação no ambiente de trabalho, a saúde ocupacional, a qualidade de vida no trabalho e a motivação dos trabalhadores como fatores que influenciam sua ocorrência. A qualidade do sono dos trabalhadores também é apontada como uma das variáveis antecedentes ao absenteísmo laboral (Kadotani, Takami, Nishikawa, Masahiro, \& Naoto, 2017; Reynolds et al., 2017).

Os impactos do absenteísmo sobre os custos organizacionais estão relacionados à contratação de trabalhadores temporários para substituírem os afastados, o pagamento extraordinário de horas para o cumprimento do cronograma de produção e o baixo rendimento dos trabalhadores que retornam de afastamentos (Palheta, 2016). Esses custos, de mensuração complexa, superam o do presenteísmo, que ocorre quando os trabalhadores exercem suas atividades profissionais mesmo em estado de adoecimento (Strömberg, Aboagye, Hagberg, Bergström, \& Lohela-Karlsson, 2017). O absenteísmo ainda é agravado pelo fenômeno da retroalimentação, na medida em que a ausência de alguns membros da organização aumenta o índice de fadiga dos trabalhadores presentes, o que aumenta a probabilidade de adoecimento e amplia a probabilidade de absenteísmo (Palmer, 2018).

Em relação aos fatores organizacionais, o estresse e a satisfação dos indivíduos estão relacionados a elevados níveis de falta ao trabalho, tendo repercussão, também, na percepção de clima organizacional (Bonatti, 2011). A carga horária (Deery, Walsh, Zatzick, \& Hayes, 2017), a insatisfação com as condições de trabalho e o relacionamento interpessoal inadequado também foram associados ao absenteísmo (Kurcgant, Passos, Oliveira, Pereira, \& Costa, 2015).

Quanto aos fatores sociodemográficos, o índice geral de desemprego de um país tem uma correlação negativa com o absenteísmo, pois o número de faltas ao trabalho tende a diminuir quando há um aumento na taxa de desemprego (Livanos \& Zangelidis, 2013; Scoppa \& Vuri, 2014). Ter filhos também está associado às faltas ao trabalho (Hohenreuther, Marconato, Souza, Lanes, \& Magnago, 2017), enquanto, no estudo de Calil, Jericó e Perroca (2015), a idade não foi apontada como antecedente.
Há ainda os fatores laborais e individuais que estão relacionados às faltas dos trabalhadores. Simões e Rocha (2014) relatam que o perfil de adoecimento associado à atividade profissional difere entre os trabalhadores que executam atividades rurais e as profissões administrativas. Já os transtornos mentais representam uma parcela dos fatores individuais que implicam em absenteísmo (Santana, Sarquis, Brev, Miranda, \& Felli, 2016), embora, em relação ao gênero dos trabalhadores (Palmer, 2018), não tenha sido encontrada qualquer evidência empírica que os associasse. Palmer (2018) ainda concluiu que o tipo de atividade profissional é a variável antecedente central que explica o absenteísmo.

Para diminuir o impacto das faltas ao trabalho nas organizações, além de identificar suas possíveis causas, é preciso ter uma política de gestão específica. Ter um programa de gestão disciplinar das faltas não justificadas, oferecer assistência às necessidades dos trabalhadores, estabelecer uma cultura que proporcione bem-estar, ter uma gestão flexível, oferecer serviço de cuidados para seus filhos além de permitir jornadas de trabalho flexíveis e bonificação aos assíduos são procedimentos que diminuem a incidência do fenômeno (Kocakulah, Kelley, Mitchell, \& Ruggieri, 2016).

Além dos impactos organizacionais, o absenteísmo também é responsável por parte das despesas do regime geral de previdência social do Brasil. Mendes, Lima e Pereira (2018) identificaram que, desde o ano de 2010, o custo anual com o absenteísmo laboral, resultante exclusivamente de adoecimento, é superior a 1,5 bilhão de reais, afetando significativamente o regime previdenciário do país.

Em relação à mensuração do impacto do absenteísmo laboral nas horas de trabalho das organizações, Marras (2005) propõe uma fórmula de cálculo que resulta no índice de controle da taxa de frequência do absenteísmo laboral, como descrito abaixo:

A partir do cálculo do índice de frequência de absenteísmo, pode-se comparar os resultados com os índices de outras organizações de porte similar ou analisar as recomendações encontradas na literatura. Uma recomendação amplamente utilizada é a de Couto (1987), que sugere que o índice considerado adequado para as organizações é $\leq 0,10$.

No campo da Psicologia, o primeiro estudo sobre a busca de evidências de validade de uma escala de absenteísmo laboral encontrada na literatura é de Paget, Lang e Shultz (1998), que dispõe de 13 itens divididos entre os fatores "voluntário" $(\alpha=0,87)$ e "involuntário" $(\alpha=0,67)$. Os itens da escala estão divididos 
entre aqueles sobre os quais os trabalhadores fazem uma escolha sobre a falta ao trabalho e aqueles que se referem a condições que independem do desejo do trabalhador e provocam sua ausência no trabalho.

Entendendo que esse é um fenômeno multicausal, Jesus e Murcho (2014) elaboraram e validaram a Escala de Fatores de Absenteísmo Laboral (EFAL) para a população portuguesa. A finalidade do instrumento, segundo os autores, é verificar a percepção dos trabalhadores a respeito dos fatores que causam mal-estar no trabalho e estão relacionados com o absenteísmo laboral. Jesus e Murcho (2014) consideram que o trabalho é uma fonte tanto de bem-estar quanto de mal-estar para os indivíduos. Esse mal-estar está relacionado às condições inadequadas de trabalho e pode ser entendido como causa ou consequência do estresse profissional, podendo também causar o desejo de abandonar a profissão e o absenteísmo. Durante o processo de validação da EFAL, a escala foi aplicada em uma amostra de 194 enfermeiros, de ambos os gêneros, que trabalhavam em hospitais e centros de saúde públicos da região do Algarve. Para as respostas, foi utilizada uma escala de frequência com seis pontos contendo as palavras "pouco" e "muito" nas extremidades.

A escala proposta por Jesus e Murcho (2014) é constituída de vinte e dois itens que estão agrupados em quatro fatores ou subescalas (como denominadas pelos autores). A consistência interna obtida para a EFAL, tomada como escala total (Jesus \& Murcho, 2104), foi de $\alpha=0,93$. Quanto ao índice de precisão de cada fator (ou subescalas) foram $\alpha=0,89$ para Fatores Interpessoais e do Ambiente de Trabalho, $\alpha=0,84$ para Fatores Psicossomáticos e de Condições de Trabalho, $\alpha=86$ para Fatores Administrativo-Laborais e $\alpha=78$ para Fatores de Gestão do Tempo e da Carreira. $\mathrm{Na}$ análise fatorial, constatou-se que os quatro fatores explicam $66,3 \%$ da variância total. Os Fatores Interpessoais e do Ambiente de Trabalho, com seis itens, referem-se ao relacionamento interpessoal no trabalho e às condições do próprio ambiente de trabalho. Os Fatores Psicossomáticos e de Condições de Trabalho estão associados aos aspectos psicoemocionais e físicos dos indivíduos e às condições ocupacionais. Os Fatores de Gestão de Tempo e da Carreira, com quatro itens, estão relacionados à gestão pessoal do tempo no trabalho e à evolução profissional na carreira. Os Fatores Administrativo-Laborais, com quatro itens, estão relacionados ao próprio trabalho e à relação que o trabalhador estabelece com a organização onde trabalha (Jesus \& Murcho, 2014).

Ao se comparar os instrumentos psicométricos validados sobre o absenteísmo com a mensuração direta por meio do cálculo de seus índices, são observadas diferenças na concepção teórica que subsidia as mensurações. Enquanto os autores que validaram as escalas (Jesus \& Murcho, 2014; Paget, Lang, \& Shultz, 1998) compreendem o absenteísmo laboral como um fenômeno psicológico que envolve a percepção de mal-estar no trabalho e desencadeia faltas e afastamentos do trabalho, os autores que relatam a mensuração por meio do cálculo baseado na frequência de faltas e afastamentos (Marras, 2005; Tonelli, 2010) entendem o absenteísmo laboral como um número bruto resultante da frequência de faltas e afastamentos que ocorre em uma organização.

Apesar de ser um tema com relevância tanto nos estudos nacionais quanto nos internacionais, o absenteísmo laboral não dispõe de muitos instrumentos psicométricos com evidências de validade, ou ainda que sejam adaptados ao contexto brasileiro. Sendo assim, este trabalho tem como objetivo a adaptação, validação de conteúdo e a busca de evidências de validade com base na estrutura interna da escala de absenteísmo laboral de Jesus e Murcho (2014), originalmente concebido para a população portuguesa, para a população brasileira.

A decisão relativa à adaptação e validação desse instrumento se deve a inconsistências identificadas no instrumento original (apesar do adequado $\alpha=0,93$ para a escala tomada como um todo), como a proposta de extração de quatro fatores e a nomenclatura utilizada. A proposta de extração de quatro fatores carece de fundamentação baseada em relatos de pesquisas ou teorias prévias ao desenvolvimento do instrumento. Além disso, os autores (Jesus \& Murcho, 2014) não apresentaram os critérios que os levaram a decidir pelo procedimento.

Outra inconsistência teórica percebida na validação da EFAL reside no relato dos autores de que "quanto maior for o valor do escore fatorial médio, maior será a percepção da importância que o respondente dará ao fator de mal-estar relacionado com o absenteísmo laboral" (Jesus \& Murcho, 2014, p. 23). O conflito ocorre porque a EFAL mensura, conforme os autores, a percepção de mal-estar no trabalho, que por sua vez é considerado um fator constituinte do absenteísmo laboral. Entretanto, Jesus e Murcho (2014) relatam que o absenteísmo laboral e a percepção de mal-estar são construtos distintos. Logo, a utilização do nome "Escala de Fatores de Absenteísmo Laboral" não seria adequada, visto que a escala mensura, na verdade, a percepção dos trabalhadores sobre mal-estar no trabalho. 
Portanto, compreende-se como necessária uma adaptação e validação do instrumento, principalmente ao se considerar que a mensuração do absenteísmo representa um dos objetivos mais antigos na área de gestão de pessoas. Adicionalmente, apesar de a frequência de estudos sobre o absenteísmo laboral ter diminuído nas últimas décadas, questões relativas a mudanças sociais, econômicas e legislativas dos últimos anos possivelmente provocaram alterações no panorama trabalhista. Considerando esse cenário de mudanças, entende-se como necessária a retomada de pesquisas em relação ao absenteísmo laboral, principalmente no que concerne à sua adequada mensuração.

\section{Método}

Este trabalho é composto por dois estudos que visaram a busca e análise de evidências de validade de conteúdo da versão brasileira da EFAL (Jesus \& Murcho, 2014), renomeada EPAAL. Ambos os estudos obtiveram autorização do Comitê de Ética em Pesquisa, como consta no parecer consubstanciado $\mathrm{n}^{\mathrm{o}} 1.206 .214$, de maneira que os participantes atuaram de forma voluntária, tiveram o sigilo preservado e registraram consentimento por meio de Termo de Compromisso Livre e Esclarecido.

Primeiramente, foram realizados os procedimentos técnicos para a adaptação semântica da escala. Em seguida, realizou-se o Estudo 1, com a primeira coleta de dados e análise fatorial exploratória. Por fim, foi realizado o Estudo 2, com uma nova coleta de dados que foram utilizados para a análise fatorial confirmatória. Tais etapas estão baseadas em sugestão de Borsa, Damásio e Bandeira (2012), mas ajustadas para a adaptação transcultural de um instrumento que já se encontra na língua para a qual se deseja adaptar. As etapas estão descritas a seguir.

\section{Adaptação Semântica do Instrumento}

Considerando que o instrumento já se encontrava na língua portuguesa, não foi necessário a tradução, e sim a adaptação ao português brasileiro. Portanto, foi realizada pelos próprios autores do presente estudo, a adaptação semântica dos termos utilizados nos itens do instrumento com a finalidade de adequar a linguagem para a população brasileira de modo que fosse mantido o significado conceitual dos itens. Por exemplo, o item "Instabilidade laboral" foi alterado para "Sinto que posso perder meu emprego a qualquer momento".
Outra alteração realizada foi a divisão do item 3 do instrumento original em outros 2 itens. No instrumento original, o item constava como "estresse e ansiedade". Como esses são dois construtos distintos, foram separados para que o instrumento esteja em concordância com a recomendação de Pasquali (2010), que aponta o critério da simplicidade para que cada item expresse apenas um construto. As demais alterações foram realizadas de modo a transformar os itens em sentenças na primeira pessoa, o que aumentaria a identificação dos respondentes com o instrumento. Na Tabela 1, há a apresentação da comparação entre os itens originais e os adaptados. Em função das alterações na grafia e número dos itens, assim como por considerar necessária a readequação ao construto mensurado pelo instrumento, a escala desenvolvida neste estudo foi intitulada "Escala de Percepção de Antecedentes do Absenteísmo Laboral" ou EPAAL.

Num segundo momento, o instrumento foi submetido ao crivo de 10 trabalhadores formalmente empregados em organizações públicas e privadas da região do Triângulo Mineiro para a verificação do nível de compreensão dos itens e das instruções de preenchimento do questionário. Os instrumentos foram entregues separadamente para cada um dos indivíduos e foi solicitado que eles realizassem apontamentos em relação à existência de dúvidas sobre a compreensão semântica do instrumento. Os participantes dessa etapa demonstraram não ter dúvidas quanto à compreensão dos itens ou em relação ao texto de instrução para o preenchimento do questionário.

Em seguida, a EPAAL foi distribuída para uma amostra de dez juízes doutores em Psicologia, que avaliaram a adequação semântica e os aspectos teóricos dos itens do questionário. No material entregue para os juízes, havia um espaço destinado à realização de críticas ou sugestões quanto à adequação semântica dos itens do instrumento. Nessa etapa, houve um índice de concordância superior a $80 \%$ em relação às questões semânticas do instrumento, por isso os itens foram mantidos como estavam.

A seguir, serão descritos os estudos conduzidos para realização do processo de busca por evidências de validade da escala adaptada. Primeiramente, apresentar-se-á o método e os resultados de cada estudo, para posteriormente realizar-se uma discussão geral acerca dos resultados encontrados.

\section{Estudo 1}

Esse primeiro estudo teve como objetivo cumprir o primeiro passo do processo de busca de evidências de 
Tabela 1.

Comparação entre o Instrumento Original e o Instrumento Adaptado

Instrumento Original

1- Insatisfação com a organização dos serviços.

2- Falta de reconhecimento profissional pelos outros (outros profissionais, clientes/doentes etc.).

3- Estresse e ansiedade.

4- Sentir-se injustiçado no serviço/instituição onde trabalha.

5- Cansaço.

6- Baixa remuneração.

7- Necessidade de tempo para formação contínua/pósgraduada.

8- Problemas com os colegas.

9- Sobrecarga de trabalho.

10- Necessidade de resolver assuntos burocráticos pessoais/ familiares inadiáveis (finanças, bancos, escola, etc.).

11- Problemas com superiores hierárquicos.

12- Trabalho por turnos.

13- Não gostar das funções desempenhadas.

14- Instabilidade laboral.

15- Realização de atividades de lazer que não podem ser realizadas durante as férias ou folgas.

16- Sentir que estagnou na carreira.

17- Mau ambiente de trabalho.

18- Desmotivação.

19- Deficientes condições físicas de trabalho.

\section{Instrumento Adaptado}

1- Me sinto insatisfeito com a organização das tarefas.

2- Não me sinto reconhecido profissionalmente pelos meus colegas.

3- Me sinto estressado.

4- Me sinto ansioso.

5- Me sinto injustiçado no serviço/instituição onde trabalho.

6- Me sinto cansado.

7- Sou mal remunerado.

8- Sinto necessidade de tempo para continuar meus estudos.

9- Tenho problema de relacionamento com meus colegas.

10- Me sinto sobrecarregado de tarefas.

11- Sinto necessidade de resolver assuntos burocráticos pessoais/familiares urgentes.

12- Tenho problemas com meu supervisor.

13- Trabalho por turnos.

14- Não gosto das funções que desempenho.

15- Sinto que posso perder o emprego a qualquer momento.

16- Me sinto impossibilitado de realizar atividades de lazer.

17- Me sinto estagnado na carreira profissional.

18- Tenho um mau ambiente de trabalho.

19 - Me sinto desmotivado.

20- Sinto que as condições físicas são inadequadas. validade do instrumento. Nessa etapa, o instrumento acima descrito foi aplicado, juntamente com o questionário sociodemográfico, visando realizar a exploração da estrutura fatorial adaptada.

\section{Participantes}

O estudo contou com uma amostra de 227 participantes, sendo 133 mulheres e 94 homens. O número de participantes definidos para a validação do instrumento foi compatível com as instruções de Pasquali (2005), que pondera que o número de participantes adequado para uma pesquisa se encontra entre cinco e dez participantes por item, cem participantes para cada fator previsto ou no mínimo duzentos participantes.
Os participantes tinham, no momento em que o questionário foi aplicado, no mínimo, 18 anos de idade, com idade média de 28 anos e desvio padrão de 8,6 anos. Além disso, o tempo médio de trabalho na empresa em que estavam empregados no momento do estudo foi de três anos e seis meses, com desvio padrão de 5,6. A amostra foi composta, em sua maioria, por participantes do sexo feminino $(58,5 \%)$, com ensino médio completo $(37,6 \%)$, solteiro $(57,7 \%)$, com idade média de 28,5 anos e 3,5 anos de trabalho. Houve indivíduos residentes de 20 estados do Brasil, além do Distrito Federal, sendo Minas Gerais, com $25,6 \%$, e São Paulo, com 18,9\%, os estados com maior frequência de participantes. 


\section{Procedimentos}

Levando-se em consideração a necessidade de aumentar a representatividade da população brasileira na amostra pesquisada, optou-se por realizar a coleta de dados virtual, uma vez que tal escolha permitiria maior heterogeneidade da amostra por abranger distintas regiões do país contempladas com respondentes da pesquisa. Para a aplicação virtual, foi inserida a escala adaptada em um formulário do aplicativo Google docs, que é gratuito. O questionário foi enviado para os potenciais respondentes por meio de uma rede de relacionamentos virtuais.

\section{Instrumentos}

Neste estudo, a primeira versão da EPAAL descrita acima foi utilizada. A escala apresentava 20 itens, diferentemente da escala original, que apresentava 19. Juntamente com a escala foi aplicado um questionário sociodemográfico, que investigou aspectos relativos às características demográficas dos participantes, como sexo, escolaridade, estado civil, idade, tempo de trabalho, tempo na organização atual.

\section{Procedimentos de Análise de Dados}

Para a realização da análise estatística dos dados, foi utilizado o software Statistical Package for the Social Sciences - SPSS 21. Verificou-se a presença de dados extremos (outliers) e de dados omissos (missing values), que não excederam 5\% em relação ao tamanho da amostra, proporção considerada ideal por Tabachnick e Fidell (2007). Os dados encontrados apresentaram a normalidade esperada, tendo como referência o índice máximo de assimetria proposto por Miles e Shevlin (2001), entre o intervalo de \pm 1 .

Para verificação da adequação da amostra, foi realizado o teste KMO (Kayser-Mayer-Olkin), sendo o valor resultante igual a 0,91, considerado adequado de acordo com as recomendações de Hair, Anderson, Tatham e Black (2009). Também foi executado o teste de Esfericidade de Bartlett's para verificar se o modelo de análise fatorial seria indicado para as variáveis e a amostra da pesquisa, sendo o resultado igual $\mathrm{x}^{2}=1917,170 ; p \leq$ 0,001, considerado adequado (Hair, Anderson, Tatham, \& Black, 2009). Uma vez atendidos os pressupostos estatísticos, os dados foram submetidos aos procedimentos indicados por Laros (2012), Pasquali (2005) e Hair et al., (2009) para a realização da análise fatorial exploratória, com o objetivo de analisar a estrutura fatorial do instrumento. Primeiramente, para identificar o número de fatores possíveis, foi executado o teste
Principal Components, com o estabelecimento padrão do programa para autovalores iguais ou maiores que 1. Em seguida, foi realizada a Principal Axis Factoring (PAF). Os resultados obtidos são descritos a seguir.

\section{Resultados}

Os resultados da análise de componentes principais demonstram a existência de quatro possíveis fatores com autovalor superior a 1. Todavia, o primeiro possível fator apresentou 38,68\% de variância explicada, enquanto os outros possíveis fatores obtiveram valores inferiores a $8 \%$ de variância explicada, o que sinaliza, a priori, a viabilidade do modelo unifatorial, conforme apontado por Vitória, Almeida e Primi (2006). Adicionalmente, foi realizado o teste Scree Plot, que apresentou o predomínio de um único fator, que alcança eigenvalue próximo a 8 e é responsável pela maior variância explicada, além de haver uma redução gradativa dos outros fatores. Tendo como referência os resultados dos testes realizados, identificou-se a possibilidade de o instrumento ser unifatorial.

Com a finalidade de obter maior precisão para a solução fatorial do instrumento, foram adotados critérios mais rígidos, com autovalor maior ou igual a 1,5 e cargas fatoriais maiores ou iguais a 0,40 , que atende a recomendação mínima Tabachnick e Fidell (2007). Então, conforme indicado por Pasquali (2005) e Hair et al., (2009), após o estabelecimento desses critérios, foi realizada a análise fatorial exploratória (Principal Axis Factoring - PAF), com rotação direct oblimin, testando a viabilidade de 1 fator. Conforme disposto na Tabela 2, identifica-se que os dois itens que obtiveram as maiores cargas fatoriais foram: a) 18 -“Tenho um mau ambiente de trabalho", com alfa igual a 0,77; b) 19 - "Me sinto desmotivado", com alfa igual a 0,81 . Os resultados apresentados na Tabela 2 explicitam a carga fatorial e as comunalidades de cada item, além do autovalor do único fator extraído e do coeficiente alfa de Cronbach do instrumento. O fator único extraído possui autovalor de 7,73 e variância explicada de 38,68\%. Os itens 8 e 13 foram excluídos do instrumento porque não atingiram a carga fatorial mínima de 0,40. Dezoito itens foram agrupados pelo fator único.

Em seguida, foram realizadas as análises para a verificação da consistência interna da escala resultante da análise fatorial exploratória. O critério utilizado foi o coeficiente alfa de Cronbach (Hair et al., 2009). O único fator extraído do instrumento obteve $\alpha=0,91$, sendo considerado bastante satisfatório (Hair et al., 2009). 
$\underline{\text { Estudo } 2}$

\section{Participantes}

O Estudo 2 contou com 202 participantes, sendo 114 mulheres e 88 homens. No momento da aplicação do questionário todos os indivíduos tinham, no mínimo, 18 anos de idade. Já a idade média dos participantes foi de 28 anos com desvio padrão de 9,6, enquanto o tempo médio de trabalho foi de três anos e sete meses com desvio padrão de 3,84. O nível de escolaridade predominante foi o ensino superior incompleto $(42,6 \%)$, seguindo-se o ensino médio completo $(36,6 \%)$, ensino superior completo $(9,4 \%)$, ensino fundamental completo $(9,4 \%)$ e pós-graduação completa $(2 \%)$. Por fim, a maioria dos participantes da amostra estava solteira $(60,9 \%)$ e a minoria, casada $(39,1 \%)$.

\section{Procedimentos}

Para a realização da análise fatorial confirmatória, os dados foram coletados presencialmente em espaços públicos da cidade de Uruaçu-GO. A opção pela coleta de dados presencial foi decorrente do baixo número de respostas obtidas na coleta de dado virtual do Estudo 1.

\section{Instrumentos}

No Estudo 2, foi utilizada a versão resultante do instrumento aplicado no Estudo 1, agora unifatorial, com 18 itens e alfa de Cronbach igual a 0,91. Adicionalmente, foi aplicado o mesmo questionário sociodemográfico, que investigava junto aos participantes informações relativas a sexo, escolaridade, estado civil, idade, tempo de trabalho e tempo na organização atual.

Tabela 2.

Cargas Fatoriais e Comunalidades dos Itens do Instrumento

\begin{tabular}{lcc}
\hline \multicolumn{1}{c}{ No meu trabalho eu... } & Carga fatorial & Comunalidades \\
\hline 1- Me sinto insatisfeito com a organização das tarefas. & 0,57 & 0,415 \\
2- Não me sinto reconhecido profissionalmente pelos meus & 0,519 & 0,492 \\
colegas. & 0,682 & 0,702 \\
3- Me sinto estressado. & 0,543 & 0,459 \\
4- Me sinto ansioso. & 0,726 & 0,618 \\
5- Me sinto injustiçado no serviço/instituição onde trabalho. & 0,671 & 0,629 \\
6- Me sinto cansado. & 0,611 & 0,425 \\
7- Sou mal remunerado. & 0,419 & 0,547 \\
9- Tenho problema de relacionamento com meus colegas. & 0,646 & 0,525 \\
10- Me sinto sobrecarregado de tarefas. & 0,495 & 0,462 \\
11- Sinto necessidade de resolver assuntos burocráticos pessoais/ & \\
familiares urgentes. & 0,568 & 0,505 \\
12- Tenho problemas com meu supervisor. & 0,538 & 0,542 \\
14- Não gosto das funções que desempenho. & 0,455 & 0,659 \\
15- Sinto que posso perder o emprego a qualquer momento. & 0,697 & 0,615 \\
16- Me sinto impossibilitado de realizar atividades de lazer. & 0,66 & 0,634 \\
17- Me sinto estagnado na carreira profissional. & 0,773 & 0,667 \\
18- Tenho um mau ambiente de trabalho. & 0,816 & 0,721 \\
19 - Me sinto desmotivado. & 0,655 & 0,601 \\
20- Sinto que as condições físicas são inadequadas. & 7,737 & \\
Autovalor & 38,683 & 0,91 \\
Variância explicada (\%) & 18 & 2 \\
Alfa de Cronbach & & \\
Números de itens adequados & & \\
Número de itens excluídos & & \\
\hline
\end{tabular}




\section{Procedimentos de Análise dos Dados}

Para a confirmação da estrutura fatorial encontrada no Estudo 1, foi realizada, nessa segunda amostra, a análise fatorial confirmatória (AFC), que consiste em uma técnica de modelação com finalidade de verificar a validade de teorias que propõem relações hipotéticas entre distintas variáveis (Marôco, 2014). Com a finalidade de definir o melhor ajuste para o modelo unifatorial proposto para a EPAAL, foi utilizado o pacote estatístico AMOS (Analysis of Moment Structuresi), versão 20. O ajustamento global do modelo fatorial foi baseado na verificação dos índices Qui-quadrado $\left(x^{2}\right)$, GFI (Goodness of it Index), CFI (Comparative Fit Index), SRMR (Standardized Root Meansquare Residual) e RMSEA (Root Meansquare Error of Approximation).

\section{Resultados}

Na Tabela 3, é possível verificar os índices de ajustamento obtidos no modelo proposto. Os coeficientes CFI e GFI estão acima de 0,85 , porém, abaixo de 0,90 , enquanto o RMSEA e o SRMR estão abaixo de 0,10. $\mathrm{O}$ ajuste do modelo demonstrou ser estatisticamente significativo.

A Figura 1 apresenta o diagrama com a representação estrutural do modelo. As cargas fatoriais mais baixas (menos de 0,40) foram encontradas nos itens 4, 9, $11 \mathrm{e}$ 15. Os itens 5, 14 e 19 apresentam as cargas fatoriais mais altas, com índices acima de 0,60. Todos os demais itens apresentaram cargas fatoriais superiores a 0,40. Considerando os coeficientes de ajuste dispostos na Tabela 3, foram observados os índices de modificação, que apontaram uma melhoria considerável do modelo diante do estabelecimento de covariâncias. Logo, foram inseridas covariâncias entre os erros dos itens 3 e 4, 3 e 6, 9 e 12

Tabela 3.

Índices de Ajustamento do Modelo Unifatorial

\begin{tabular}{cc}
\hline Índice de ajustamento & Valor obtido \\
\hline$x^{2}-$ Qui-quadrado & $260,7^{*}$ \\
GFI & $0,87^{*}$ \\
CFI & $0,86^{*}$ \\
RMSEA & $0,07^{*}$ \\
SRMR & $0,06^{*}$ \\
\hline
\end{tabular}

Nota. ${ }^{*} p<0,001$. e 7 e 20, uma vez que a covariância dos resíduos não prejudica a relação estabelecida entre os itens e a variável latente. Com relação aos índices de ajuste obtidos, de forma geral, a literatura indica que valores de RMSEA entre 0,05 e 0,08 são aceitáveis (e.g., Fabrigar et al., 1999; Hu \& Bentler, 1999). O valor de RMSEA de 0,07 nessa amostra representa um bom ajuste para autores, como Marôco (2010) e Byrne (2001). Para Kline (2005) e Hu e Bentler (1999), valores de SRMR abaixo de 0,10 são satisfatórios, de maneira que a amostra apresentou valor igual a 0,06 , atendendo ao critério. Por outro lado, os valores de GFI e CFI estão abaixo de 0,90 . São considerados bons valores aqueles acima de 0,90 (e.g., Kline, 2005)". Para Marôco (2014), índices entre 0,80 e 0,90 indicam um ajustamento razoável enquanto outros autores defendem que valores abaixo de 0,90 simplesmente sugerem que o modelo pode ser aprimorado (Carvalho \& Chima, 2014). Por fim, há indicações na literatura de que o GFI é afetado pelo tamanho da amostra (Ainur, Sayang, Jannoo, \& Yap, 2017), que, no caso deste estudo, é considerada reduzida.

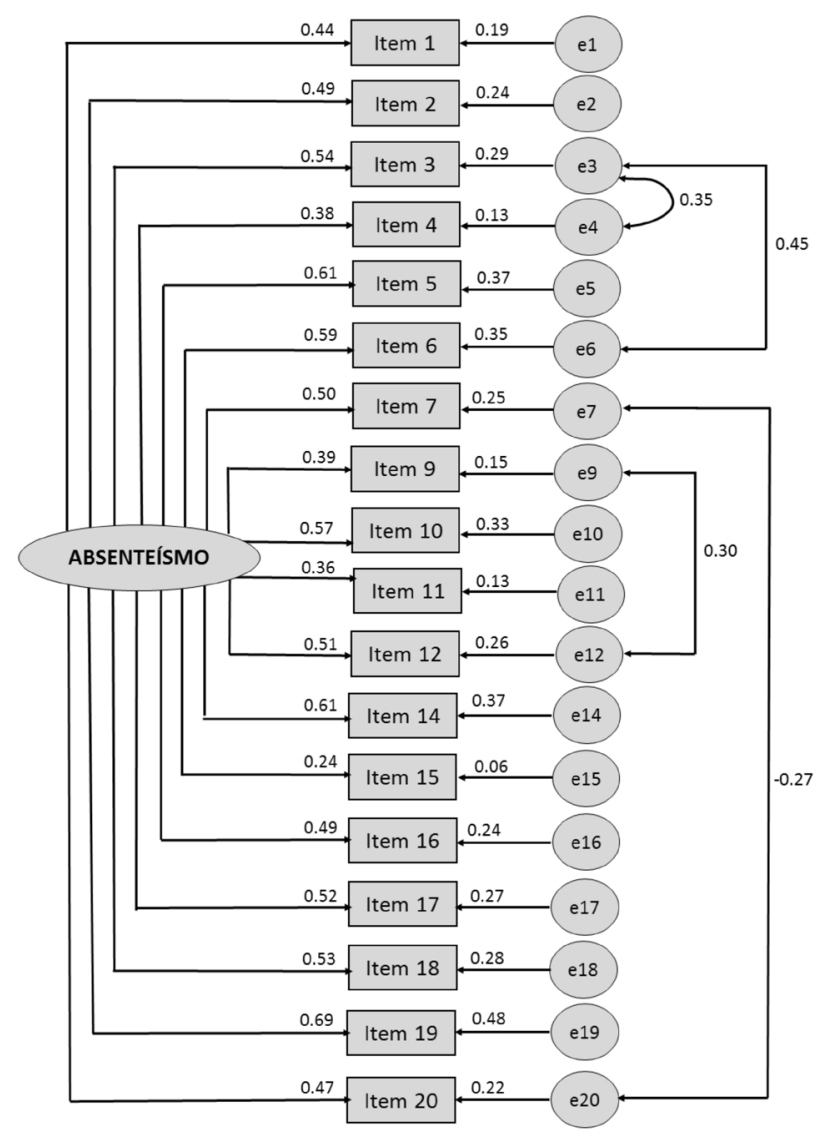

Figura 1. Modelo ajustado $(\phi \leq 0,001)$

Psico-USF, Bragança Paulista, v. 25, n. 2, p. 357-369, abr./jun. 2020 


\section{Discussão}

Os objetivos dos dois estudos aqui apresentados foram atingidos, uma vez que a EPAAL passou tanto pelo processo de análise exploratória quanto confirmatória, sendo possível constatar alterações consideráveis na estrutura fatorial da versão brasileira da escala. A realização de ambos estudos foi necessária devido ao fato de que foi identificada, no Estudo 1, a necessidade de mudanças estruturais no instrumento original, sendo necessária a reaplicação da escala no Estudo 2 e a confirmação da sua estrutura fatorial.

Com relação ao Estudo 1, os dois itens que obtiveram as maiores cargas fatoriais foram " 18 - Tenho um mau ambiente de trabalho", com 0,77, e "19 - Me sinto desmotivado", com 0,81. Sobre o ambiente de trabalho, Bonatti (2011) verificou que há uma relação entre a percepção de clima organizacional por parte dos trabalhadores e o absenteísmo laboral, o que pode explicar a carga fatorial alta no iem 18. Sobre a motivação, a elevada carga fatorial converge com estudos anteriores, como o de Hackman e Oldham (1976) que verificou uma baixa correlação entre a motivação dos trabalhadores e o absenteísmo laboral.

Já o resultado obtido nesta pesquisa, de um fator extraído, não foi congruente com o resultado do estudo original, no qual foram extraídos quatro fatores. Porém, ao se analisar as nomeações dos fatores propostas pelos autores (Jesus \& Murcho, 2014), pode ser constatada grande similitude entre elas. Um exemplo da similitude observada entre os fatores do instrumento original (Jesus \& Murcho, 2014) ocorre entre o item "17 - Mau ambiente de trabalho" e o item "19 - Deficientes condições físicas de trabalho". O item 17 foi categorizado nos "fatores interpessoais e do ambiente de trabalho" e o item 19 foi categorizado nos "fatores psicossomáticos e de condições de trabalho". Os próprios itens resguardam uma semelhança conceitual, pois as condições físicas de trabalho são componentes do próprio ambiente de trabalho. Além disso, os autores não propuseram qualquer distinção teórica entre os termos referidos nos fatores do instrumento, o que pode denotar uma limitação teórica.

Considerando os resultados obtidos no Estudo 2, a questão da similitude entre itens é observada no estabelecimento de covariâncias. Os itens 3 e 6, por exemplo, questionam os respondentes sobre estresse, ansiedade e cansaço, conceitos que rotineiramente são dispostos como elementos associados. Entre o item 9 (Tenho problema de relacionamento com meus colegas) e o item 12 (Tenho problemas com meu supervisor) também foi identificada uma alta covariância, que pode ser explicada pelo fato de que ambos (colegas e supervisor) embora com status diferenciado, constituem relações interpessoais no trabalho que, pelo que indicam os resultados, são percebidos como equivalentes. Também há uma covariância entre os resíduos dos itens 7 (Sou mal remunerado) e do item 20 (Sinto que as condições físicas são inadequadas). Nesse caso, compreende-se que ambos os itens se referem a condições prévias adequadas (quando presentes) para índices aceitáveis de absenteísmo e dizem respeito, ambos, a condições organizacionais de trabalho. Finalmente, considerando o conjunto dos índices de ajuste, a tendência à unifatoralidade e a coerência teórica do modelo, é possível considerá-lo aceitável, podendo ser aprimorado por meio de uma coleta de dados mais ampla no futuro.

Devido ao fato de a dimensão latente do construto ser a percepção sobre o mal-estar no trabalho, o fator extraído foi compreendido como fator de mal-estar no trabalho. Embora haja, na literatura, diferentes concepções teóricas do absenteísmo laboral, este estudo partiu da concepção psicológica do construto, concebendo-o como uma construção latente antecedente à intenção de deixar a organização empregadora ou ao ato em si. A construção do instrumento original (Jesus \& Murcho, 2014), embora se referisse a fatores constituintes do absenteísmo, versava, conforme os próprios autores, a situações ou condições que, se e quando presentes, poderiam levar o empregado deixar o sistema empregador. Ou seja, a concepção teórica assumida pelos autores deste estudo está congruente com o estudo original, sendo esse um dos motivos que levaram ao trabalho de adaptação empreendido, além, é claro, da necessidade de adaptação semântica que capacitasse a utilização do instrumento a contextos brasileiros.

A respeito da dimensão unifatorial, há o entendimento de que o absenté́smo laboral possui uma dimensão latente e uma manifestação comportamental. Pensando em termos de atitude, o aspecto cognitivo seria a percepção dos trabalhadores sobre o mal-estar no trabalho, que geraria uma tendência às faltas. Já a manifestação comportamental do fator se caracteriza pela falta ou o afastamento do trabalho, e é ela que pode ser diretamente observada pela gestão da organização por meio do número bruto de faltas.

Logo, uma vez que a mensuração da escala oferece uma estimativa sobre as variáveis antecedentes ao fenômeno, e não a mensuração dos fatores componentes do absenteísmo laboral em si, a nomenclatura 
original do instrumento não se apresenta congruente com o objeto mensurado. Por isso, o nome proposto para o instrumento foi "Escala de Percepção de Antecedentes do Absenteísmo Laboral" - EPAAL, devendo ser utilizada para diagnósticos sobre a percepção dos trabalhadores a respeito de variáveis que antecedem o absenteísmo no trabalho.

Além dos aspectos citados, sobre a similitude entre os fatores do instrumento original e em relação aos itens de fatores distintos, Jesus e Murcho (2014) relatam que os itens da escala mensuram a percepção dos trabalhadores sobre variáveis relacionadas ao mal-estar no trabalho, que, por sua vez, é um antecedente do absenteísmo laboral. Além disso, tendo como referência o instrumento validado por Paget et al., (1998), a percepção de mal-estar no trabalho pode ser entendida como uma variável latente antecedente às faltas ou aos afastamentos do trabalho. Tal como proposto por Paget et al., (1998), o fator extraído do instrumento validado nesta pesquisa é entendido como a percepção dos trabalhadores sobre o mal-estar no trabalho que, por sua vez, parece se constituir um antecedente de absenteísmo laboral e não seu constituinte.

\section{Considerações Finais}

Diante da importância do absenteísmo laboral para a análise do comportamento e dos resultados organizacionais e da existência de poucos instrumentos de mensuração do fenômeno, este trabalho teve como objetivo a adaptação transcultural e a busca por evidências de validade de uma escala de absenteísmo laboral para o ambiente brasileiro. As análises realizadas resultaram em um instrumento fidedigno, adaptado para o contexto brasileiro e com índices psicométricos confiáveis. Embora os índices de ajuste do modelo possam ser aprimorados com novos estudos, de forma geral o instrumento pode vir a ser utilizado tanto para fins diagnósticos quanto em investigações de cunho acadêmico.

Sobre as limitações deste estudo, a primeira se refere à amostra da segunda coleta de dados. Enquanto na primeira houve a tentativa de preservar a maior variabilidade da amostra, utilizando um formulário virtual e contando com indivíduos residentes de distintos estados do Brasil, a segunda amostra contou com membros residentes de apenas uma cidade. Tal fato pode ter incitado maior homogeneidade da amostra, o que não é desejável, pois, segundo Pasquali (2010), resultados mais precisos são garantidos pela maior variabilidade da amostra. O embasamento dos resultados exclusivamente em um questionário de autorrelato também é entendido como uma limitação, visto que uma série de variáveis pode interferir nas respostas dos participantes. Desse modo, o mais adequado seria complementar os dados obtidos por meio de questionário com informações documentais das organizações, por exemplo, visando aumentar o nível de confiabilidade das respostas.

A própria inconsistência teórica em relação à conceitualização do absenteísmo laboral também se apresentou como uma limitação do estudo, visto que a natureza da variável e a sua descrição comportamental são fundamentais para o estabelecimento de um delineamento adequado de pesquisa. Como as descrições da variável e suas formas de mensuração são despadronizadas, há um prejuízo para a comparação de resultados e a generalização dos resultados de pesquisas sobre esse tema.

Como agenda para futuras pesquisas no Brasil, sugere-se verificar qual a relação entre a percepção de mal-estar no trabalho e os índices de absenteísmo laboral dos trabalhadores. Além disso, há uma carência de pesquisas longitudinais sobre o absenteísmo laboral e de temas como a relação entre o absenteísmo laboral e a legislação trabalhista, o índice de desemprego geral e as condições locais de infraestrutura, visto que não foram encontrados estudos nacionais sobre tais temas. Ademais, a maioria dos estudos sobre o tema encontrados na literatura nacional contam com amostras compostas exclusivamente por servidores públicos, o que dificulta a generalização dos resultados.

\section{Referências}

Ainur, A. K., Deni, S. M., Jannoo, Z., \& Yap, B. W. (2017). Sample sizeand non-normality effects on goodness of fit measures in structural equation models. Pertanika Journal of Science and Technology, 25(2), 575586. Recuperado de https://uitm.pure.elsevier. $\mathrm{com} / \mathrm{en} /$ publications/sample-size-and-non-normality-effects-on-goodness-of-fit-measures

Bonatti, C. L. (2011). Infuência da força do clima sobre o absenteísmo (Dissertação de mestrado). Universidade de Brasília. Recuperado de http://repositorio.unb. br/handle/10482/10209

Borsa, J. C., Damásio, B. F., \& Bandeira, D. R. (2012). Adaptação e validação de instrumentos psicológicos. Paidéia, 22(53), 423-432. doi:10.1590/ S0103-863X2012000300014

Psico-USF, Bragança Paulista, v. 25, n. 2, p. 357-369, abr./jun. 2020 
Byrne, B. M. (2001). Multivariate applications book series. Structural equation modeling with AMOS: Basic concepts, applications, and programming. Mahwah, NJ, US: Lawrence Erlbaum Associates Publishers.

Calheiros, P. R., \& Rodrigues, E. C. (2010). Psicologia organizacional e as ferramentas usadas no enfrentamento do absenteísmo. Revista de Psicologia da IMED, 2(2), 433-440. doi:10.18256/2175-5027/ psico-imed.v2n2p433-440

Calil, A. S. G., Jericó, M. C., \& Perroca, M.G. (2015). Gerenciamento de recursos humanos em enfermagem: Estudo da interface idade - absenteísmo. Revista Mineira de Enfermagem. 19(2), 79-92. doi:10.5935/1415-2762.20150027

Carvalho, J., \& Chima, F. O. (2014). Applications of structural equation modeling in social sciences research. American International Journal of Contemporary Research, 4(1), 6-11. Recuperado de http:// www.aijcrnet.com/journals/Vol_4_No_1_January_2014/2.pdf

Couto, H. A. (1987). Temas de saúde ocupacional: Coletânea dos cadernos. Belo Horizonte: ERGO.

Deery, S., Walsh, J., Zatzick, C. D., \& Hayes, A. F. (2017). Exploring the relationship between compressed work hours satisfaction and absenteeism in front-line service work. European Journal of Work and Organizational Psychology, 26, 42-52. doi:10.1080 /1359432X.2016.1197907

Fabrigar, L. R., Wegener, D. T., MacCallum, R. C., \& Strahan, E. J. (1999). Evaluating the use of exploratory factor analysis in psychological research. Psychological Methods, 4(3), 272-299. doi:10.1037/1082-989X.4.3.272

Hackman, J. R., \& Oldham, G. R. (1976). Motivation through the design of work: Test of a theory. Organizational Behavior and Human Performance, 16(2), 250-279. doi:10.1016/0030-5073(76)90016-7

Hair, J. F., Anderson, R. E., Tatham, R. L., \& Black, W. C. (2009). Análise multivariada de dados (6 $6^{\mathrm{a}} \mathrm{ed}$.). Porto Alegre: Bookman.

Hohenreuther, R., Rodrigues, F. C. P., Marconato, C. S., Souza, M. M., Lanes, T. C., \& Magnago, T. S. B. S. (2017) Serviço hospitalar de limpeza e absenteísmo - doença no trabalho. Revista Mineira de Enfermagem, 21(1), e1055. doi:10.5935/1415-2762.20170065
Hu, L., \& Bentler, P. M. (1999). Cut off criteria for fit indexes in covariance structure analysis: Convention alcriteria versus new alternatives. Structural Equation Modeling, 6(1), 1-55. doi:10.1080/10705519909540118

Jesus, S. N., \& Murcho, N. A. (2014). Absenteísmo no trabalho. Em M. M. Siqueira (Ed.), Novas Medidas do Comportamento Organizacional - Ferramentas de Diagnóstico e de Gestão (pp. 15-24). Porto Alegre: Artmed.

Kadotani, H., Takami, M., Nishikawa, K., Masahiro, M., \& Naoto, Y. (2017). Relationship between absenteeism/presenteeism and weekday sleep debt in government employees of a Japanese city. Sleep Medicine, 40(1), e152. doi:10.1016/j. sleep.2017.11.444.

Kline, R. B. (2005). Methodology in the social sciences. Principles and practice of structural equation modeling $\left(2^{\mathrm{a}} \mathrm{ed}\right.$.). New York, NY, US: Guilford Press.

Kocakulah, M. C., Kelley, A. G., Mitchell, K. M., \& Ruggieri, M. P. (2016). Absenteeism problems and costs: Causes, effects and cures. International Business \& Economics Research Journal (IBER), 15(3), 89-96. doi:10.19030/iber.v15i3.9673

Kurcgant, P., Passos, A. R., Oliveira, J. M. L., Pereira, I. M., \& Costa, T. F. (2015). Absenteísmo do pessoal de enfermagem: Decisões e ações de enfermeiros gerentes. Revista da Escola de Enfermagem da USP [online], 49(2), 35-41. doi:10.1590/ S0080-623420150000800005

Laros, J. A. (2012). O uso da análise fatorial: Algumas diretrizes para pesquisadores. Em L. Pasquali (Org.), Análise fatorial para pesquisadores (pp. 141160). Brasília: LabPAM Saber e Tecnologia.

Livanos, I., \& Zangelidis, A. (2013). Unemployment, labor market flexibility, and absenteeism: A paneuropean study. Industrial Relations, 52(2), 492-515. doi:10.1111/irel.12021

Marôco, J. (2014). Análise de equações estruturais: Fundamentos teóricos, software \& aplicações ( $2^{\mathrm{a}}$ ed.). Lisboa: Report Number.

Marras, J. P. (2005). Administração de Recursos Humanos: Do operacional ao estratégico. São Paulo: Futura.

Mendes, N. C. F., Lima, D. V., \& Matias-Pereira, J. (2018) O impacto do absenteísmo-doença nas despesas do regime geral de previdência social do Brasil. 
Revista Eletrônica de Estratégia \& Negócios, 11(1), 100121. doi:10.19177/reen.v11e02018101-121

Miles, J., \& Shevlin, M. (2001). Applying regression and correlation: A guide for students and researches. Sage Publications: London.

OIT - Organización Internacional del Trabaho (1989). Absentismo: Causa y control. Enciclopedia de Salud e Seguridaden El Trabajo. Madrid: OIT.

Paget, K. J., Lang, D. L., \& Shultz, K. S. (1998). Development and validation of an employee absenteeism scale. Psychological Reports, 82(3), 11441146. doi:10.2466/pr0.1998.82.3c.1144

Palheta, C. A. F. (2016). Estudo do absenteísmo por doenca entre trabalhadores de uma indústria do Estado do Amazonas (Dissertação de mestrado). Universidade Federal do Amazonas, Manaus. Recuperado de http://tede.ufam.edu.br/handle/tede/5150.

Palmer, K. (2018). The Heavy Cost of Care: Systemic Challenges in Norwegian Work Absenteeism. Social Sciences, 7(6), 1-17. doi:10.3390/socsci7060094

Pasquali, L. (2005). Análise Fatorial para Pesquisadores. Brasilia: LabPAM.

Pasquali, L. (2010). Instrumentação psicológica: Fundamentos e práticas. Porto Alegre: Artmed.

Reynolds, A. C., Appleton, S. L., Gill, T.K., Taylor, A. W., McEvoy, R. D., Ferguson, S. A., \& Adams, R. J. (2017) Sickness absenteeism is associated with sleep problems independent of sleep disorders: Results of the 2016 Sleep Health Foundation national survey. Sleep Healph, 3(5), 357-361. doi:10.1016/j. sleh.2017.06.003

Santana, L. L., Sarquis, L. M. M., Brev, C., Miranda, F. M. D., \& Felli, V. E. A. (2016). Absenteísmo por transtornos mentais em trabalhadores de saúde em um hospital no sul do Brasil. Revista Gaúcha de Enfermagem, 37(1), e53485. doi:10.1590/1983- 1447.2016.01.53485

Scoppa, V., \& Vuri, D. (2014). Absenteeism, unemployment and employment protection legislation: evidence from Italy. IZA Discussion Paper, 3(3), 2-35. doi: 10.1186/2193-8997-3-3

Simões, M. R. L., \& Rocha, A. M. (2014). Absenteísmo-doença entre trabalhadores de uma empresa florestal no Estado de Minas Gerais, Brasil. Revista Brasileira de Saúde Ocupacional, 39(129), 17-25. doi:10.1590/0303-7657000061112

Strömberg, C., Aboagye, E., Hagberg, J., Bergström, G., \& Lohela-Karlsson, M. (2017). Estimating the effect and economic impact of absenteeism, presenteeism, and work environment - Related problems on reductions in productivity from a managerial perspective. Value Health, 20(8), 10581064. doi:10.1016/j.jval.2017.05.008

Tabachnick, B. G., \& Fidell, L. S. (2013). Using multivariate statistics ( $6^{\mathrm{a}}$ ed.). Boston: Pearson.

Tonelli, D. (2010). Perfil do absenteísmo-doença em indústria do interior paulista, 2007 a 2009 (Dissertação de mestrado). Universidade Estadual Paulista, Botucatu. Recuperado de http://repositorio.unesp.br/ handle/11449/96450/

Vitória, F., Almeida, L. S., \& Primi, R. (2006). Unidimensionalidade em testes psicológicos: Conceito, estratégias e dificuldades na sua avaliação. Revista de Psicologia da Vetor Editora, 7(1), 1-7. Recuperado de http://pepsic.bvsalud.org/scielo.php?script=sci_arttext\&pid=S1676-73142006000100002

Recebido em: 13/02/2018 Reformulado em: 24/02/2019 
Sobre os autores:

Sinésio Gomide Junior é graduado em Formação de Psicólogo pela Universidade de Brasília (1982), mestre em Psicologia pela Universidade de Brasília (1986) e doutor em Psicologia pela Universidade de Brasília (1999). Atualmente é professor titular da Universidade Federal de Uberlândia e membro do Grupo de Trabalho "Cultura Organizacional e Saúde no Trabalho” da Associação Nacional de Pós-Graduação em Psicologia (ANPEPP).

ORCID: https://orcid.org/0000-0003-0944-4380

E-mail: sinesiogomide@uol.com.br

Marco Aurélio Silva Esteves é graduado em Formação de Psicólogo pela Universidade Federal de Uberlândia (UFU), mestre em Psicologia Aplicada pela Universidade Federal de Uberlândia (UFU) e, atualmente, é professor e Coordenador de Apoio ao Estudante na Faculdade Serra da Mesa (FaSeM).

ORCID: https://orcid.org/0000-0003-4022-9689

E-mail: marcoestevespsi@gmail.com

Lígia Carolina Oliveira Silva é doutora e mestre pelo Programa de Pós-Graduação em Psicologia Social, do Trabalho e das Organizações (PSTO) da Universidade de Brasília - UnB, professora adjunta de Psicologia Organizacional e do Trabalho e Gestão de Pessoas na Universidade Federal de Uberlândia - UFU e coordenadora do Grupo de Pesquisa em Realização Profissional e Carreira (CNPq).

ORCID: https://orcid.org/0000-0002-7487-9420

E-mail: ligiacarol1987@gmail.com

Contato com os autores:

Caixa Postal: Av. Pará, 1720, Bloco 2C, Sala 31, Bairro Umuarama Campus Umuarama

Uberlândia-MG, Brasil

CEP: 38400-902 
